\title{
SOFT VS HARD CHIN CUP EFFECTS IN MANAGEMENT OF CLASS III MALOCCLUSIONS. A RANDOMIZED CONTROL CLINICAL TRIAL
}

\author{
Essam Abdelalim Nassar," Ahmad Mohammad Hafez ${ }^{* *}$ and Yasser Lotfy Abdelnaby ${ }^{* * *}$
}

\begin{abstract}
Objective: This study was conducted to evaluate the dento-skeletal effects of hard and soft chin cup in treatment of class III cases. Also, their effect on the skin of the chin was assessed.

Methods: The sample consisted of fifty five growing children and was randomly allocated into three groups. In group $1(\mathrm{n}=22)$ hard chin cup applying $300 \mathrm{~g}$ force/side was used, group 2 $(n=22)$ soft chin cup was utilized, while in group $3(n=11)$ no treatment was performed. Lateral cephalograms were taken for all participants before and after 12 months of treatment. The skin of the chin was evaluated using Skin Irritation Index (SII). Cephalometric data was statistically analyzed using one-way ANOVA and Tukey test while Chi-square test was utilized to evaluate the SII.

Results: There were significant reduction in SNB, 1-MP angles and Ar-Go and an increase in the ANB, SN-MP angles, Wits appraisal and N-Me measurements in the treatment groups compared to control group $(\mathrm{P} \leq 0.05)$. In addition, 1-MP angle was significantly decreased with soft chin cup than the hard chin cup $(\mathrm{P} \leq 0.05)$. Erythema of the skin was the common effect for both chin cup type. There was no significant differences between hard and soft chin cup $(\mathrm{X} 2=2.59, \mathrm{P}=0.28)$ although laceration was grater with hard chin cup.
\end{abstract}

Conclusions: Hard and soft chin cups are effective in treatment of class III malocclusions. Erythema of the chin skin was the main effect of both types and lacerations were greater with hard chin cup.

KEY WORDS: Class III treatment, Chin cup, Skin irritations.

\section{INTRODUCTION}

Class III malocclusion can result from a deficient maxilla which accounts for $18 \%$ of the recorded cases, excessive mandible that represents the majority of cases $(52 \%)$ or both.$^{1-3}$ It is also characterized by maxillary teeth protrusion and mandibular teeth retrusion ${ }^{4,5} \mathrm{~A}$ high prevalence of class III $23 \%$ is found in Asian population ${ }^{6,7}$ while a lesser percentage (5\%) exist among Caucasians. ${ }^{8,9}$

Various treatment protocols have been introduced for class III treatment based on the etiological factor. Maxillary protraction ${ }^{10,11}$ and

\footnotetext{
* Associate Professor, Department of Orthodontics, Faculty of Dentistry, Mansoura University, Mansoura, Egypt.

** Lecturer, Department of Orthodontics, Faculty of Dentistry, Mansoura University, Mansoura, Egypt.

*** Professor, Department of Orthodontics, Faculty of Dentistry, Mansoura University, Mansoura, Egypt.
} 
functional appliances ${ }^{12,13}$ were advocated for those having a maxillary deficiency. While chin cup is the treatment choice for mandibular excess patients. ${ }^{14,15}$

Chin cup is considered a popular choice since its utilization in the $19^{\text {th }}$ century till now. The rationale behind its use is to improve the anteroposterior relationship between maxilla and mandible through applying force on the mandible to restrain its growth. A downward and backward rotation of the mandible, mandibular growth inhibition, condylar growth restriction or redirection, temporomandibular joint remodeling, a decrease of gonial angle, and lingual tipping of the lower incisors are the frequent finding with the chin cup. ${ }^{16,17}$ Stability after chin cup treatment depends on many factors, such as genetic influence, growth, age, family history and patient cooperation. ${ }^{18}$

Meanwhile, there are a lot of controversies among the chip cup usage such as early treatment or late with a wide range of 5-13 years ${ }^{19}$, amount of force light versus heavy ${ }^{20}$, wearing time ranging from 8 to 18 hours ${ }^{15,17}$ as well as the utilization of chin cup alone or in conjunction with other appliances such as inclined plane ${ }^{10}$ or maxillary expansion..$^{21-23}$

Two types of chin cups are available; hard and soft. The hard one is rigid made of polyamide, padded and air permeable. Soft chin cup is elastic, made of synthetic cloth, porous and air permeable. However insufficient data in the literature are available comparing their effects on the underlying structures. Therefore the aim of this study was to compare the dental and skeletal effects of the hard or soft chin cups in class III patients and evaluating their effects on the underneath chin skin.

\section{MATERIAL AND METHODS}

\section{Study population}

This trial design was a 3 parallel arms randomized active controlled clinical trial with the ratio group1: group2: group 3 (control) $=2: 2: 1$. No changes were done to the trial after commencing. The following inclusion criteria were applied: growing patients with skeletal Class III malocclusion (ANB angle less than 1 degree) due to mandibular excess (SNB angle $\geq 80$ degrees), SN-MP $=35+2$ degrees and an anterior crossbite. Exclusion criteria included extracted, missing, supernumerary teeth, previous orthodontic treatment, temporomandibular joint dysfunction and craniofacial anomalies. Sample size was calculated through (http://powerandsamplesize. com/Calculators/Compare-2-Means/2-SampleEquality) at alpha error $=1 \%$ and study power $=90 \%$ indicated eighteen patients in groups $2 \& 3$ and 9 patients in control group. This number was increased by $20 \%$ to make up for loss through follow-up over one year period. Thus, the numbers in the group 1 , 2 and 3 were 22,22 , and 11 with a total sample size of 55 (29 boys and 26 girls). Skeletal status was evaluated through hand wrist x-ray and confirming that all patients were in the cap stage and the crest of pubertal growth spurt did not elapse. Randomization was accomplished by using the "Random Allocation Software" (http://mahmoodsaghaei.tripod.com/ Softwares/randalloc.html\# Random Allocation Software). The allocation sequence was concealed with sequentially numbered, opaque, sealed and stapled envelopes that kept with a research assistant who was not involved in treatment and measuring the outcome. At the beginning, thirty-three patients were allocated into 3 equal groups of 11 each. Subsequently, twenty-two patients were allocated to groups 1 and 2 . Twenty-two patients (12 boys and 10 girls) represented group 1 while group 2 contained 22 patients (11 girls and 11 boys), and group 3 involved 11 patients ( 6 boys and 5 girls). After the patient was assigned in the trial, the research assistant opened the next consecutive opaque envelop and revealed the treatment allocation to the operator. All patients and their parents are informed about the study and agreed to participate.

\section{Chin cup application}

At the beginning of treatment, the mean ages for children in group 1, 2 and 3 were 10.4, 9.6, and 10.1 years respectively. A hard chin cup (744-61300 , Dentaurum, Ispringen, Germany) with short hooks was utilized in group 1 , while a soft chin 
cup (744-614-00, Dentaurum, Ispringen, Germany) was used for children in group 2. All patients in groups 1 and 2 received an acrylic bite plane on the lower posterior teeth to free the occlusion. A $300 \mathrm{~g}$ force per side in occipital direction was applied for all participant and was monitored by a force gauge (Correx Co, Bern, Switzerland). A minimum of 14 hours /day appliance's wearing time was requested for all patients. Group 3 represented the control group where no treatment was done. These patients will receive another treatment later. After three months of treatment two boys dropped out from the study, one from group 1 (moved to another city) and the other one was from group 2 (cooperation reason). The final number for analysis was twentyone patients in group 1 and 2.

\section{Outcome measurement}

Lateral cephalograms were obtained for all children in a centric occlusion position before the treatment and after 12 month treatment period. Film magnifications were standardized at $8 \%$. Then cephalometric films were traced. Landmarks and measurements used in this study to evaluate skeletal and dental changes are illustrated (Figure 1)..$^{20,24}$ Thirty randomly selected cephalometric films were retraced after 20 days to assess the method errors. All the tracings were done with the second investigator who was not aware of treatment allocation.

\section{Skin Irritation Index determination}

Clinically, the skin of the chin for all patients in all groups was evaluated during the treatment period for any erythema or laceration using SII. It was given a score from 0 to 2 . A score 0 indicated no erythema (redness of skin) or laceration (disruption of skin). ${ }^{25}$ Score 1 was given if there was erythema, and score 2 if there was any laceration. The skin was evaluated by a third investigator who also was blind to the treatment allocation.

\section{Statistical Analysis:}

Statistical analysis of the data was carried out using SPSS 20 (Chicago, IL, USA).

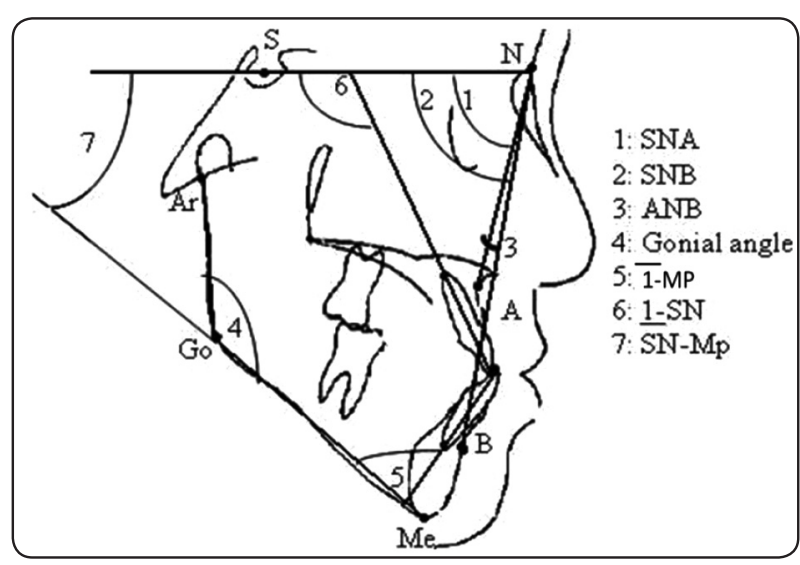

Fig. (1) Cephalometric points and measurements.

Dahlberg's formula was applied to assess the method errors. One way Analysis of Variance (ANOVA) and Tukey test was utilized to detect significant between-group differences for all cephalometric variables. Chi-square test was utilized to evaluate the SII for the studied groups. All results are significant at $P \leq 0.05$.

\section{RESULTS}

There was no significant difference between the first and second tracings of the same film and the method errors were less than $1 \mathrm{~mm}$ and $1^{\circ}$ for both linear and angular measurements. Clinically, both hard and soft chin cup usage resulted in anterior cross bite correction in all treated patients (Figure 2).

Means and standard deviations of the pretreatment cephalometric measurements in the three studied groups were presented in Table 1 while those of post-treatment cephalometric changes are presented in Table 2. ANOVA test revealed there were no significant differences in post-treatment cephalometric changes between the three studied groups in SNA, Ar-Me, Go-Me, ArGo-Me and 1 -SN measurements $(P \geq 0.05)$ while there was a significant difference between the other measurements $(P \leq 0.05)$. According to Tukey test, there was a significant reduction in SNB, 1-MP angles and Ar-Go and a significant increase in the ANB, SN-MP angles, Wits appraisal as well as N-Me measurements in the treatment groups compared to 


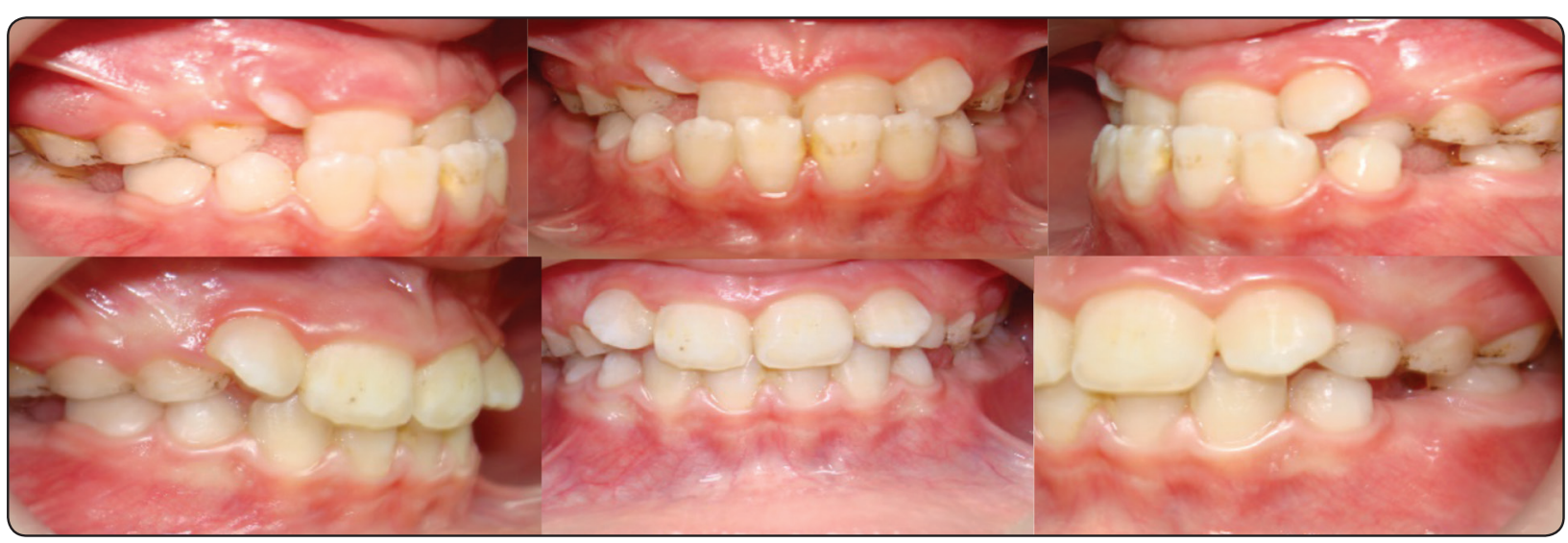

Fig. (2) Pre and posttreatment intraoral photographs of patient utilized chin cup

non-treated one $(P \leq 0.05)$. In addition, the decrease in 1-MP angle was significantly greater in soft chin cup group than the hard chin cup group $(P \leq 0.05)$.

Regarding the effect of chin cup type on the skin of the chin, Chi-square test (Table 3 ) revealed significant differences between the three studied groups $\left(\mathrm{X}^{2}=28.8, P<0.001\right)$. Additionally, no significant difference was found between hard and soft chin cup $\mathrm{p}=0.34$. However, laceration was greater in the hard chin cup group (Figure 3). On the other hand, erythema was the dominant effect in soft chin cup group $\left(\mathrm{X}^{2}=8, P=0.018\right)$.

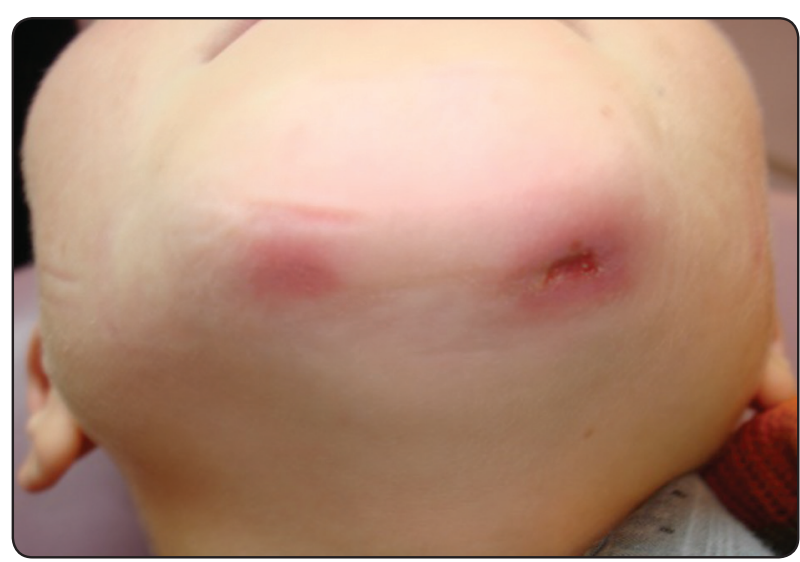

Fig. (3) Laceration in skin underneath the chin cup.

TABLE (1) Means and standard deviations (SD) of the pretreatment cephalometric measurements for the three studied groups.

\begin{tabular}{|c|c|c|c|}
\hline \multirow{2}{*}{ Measurements } & $\begin{array}{c}\text { Group 1 } \\
\text { (Hard chin cup) }\end{array}$ & $\begin{array}{c}\text { Group 2 } \\
\text { (Soft chin cup) }\end{array}$ & $\begin{array}{c}\text { Group 3 } \\
\text { (control) }\end{array}$ \\
\cline { 2 - 4 } & Means \pm SD & Means \pm SD & Means \pm SD \\
\hline SNA & $81.33 \pm 0.65$ & $81.33 \pm 0.57$ & $82.09 \pm 0.70$ \\
\hline SNB & $80.95 \pm 0.49$ & $81.04 \pm 0.66$ & $0.63 \pm 0.50$ \\
\hline ANB & $0.38 \pm 0.74$ & $0.52 \pm 0.81$ & $-6.27 \pm 1.0$ \\
\hline Wits & $-5.85 \pm 0.72$ & $-6.28 \pm 0.78$ & $93.09 \pm 2.34$ \\
\hline Ar-Me & $91.04 \pm 2.22$ & $90.28 \pm 2.28$ & $37.09 \pm 1.13$ \\
\hline Ar-Go & $36.76 \pm 1.09$ & $35.95 \pm 1.35$ & $66.81 \pm 1.47$ \\
\hline Go-Me & $66.28 \pm 1.73$ & $65.47 \pm 1.50$ & $127.63 \pm 1.56$ \\
\hline Ar-Go-Me & $128.95 \pm 2.31$ & $129.85 \pm 2.45$ & $116.90 \pm 2.07$ \\
\hline N-Me & $115.90 \pm 2.50$ & $116.47 \pm 2.46$ & $34.54 \pm 1.21$ \\
\hline SN-MP & $34.66 \pm .91$ & $35.04 \pm 1.07$ & $98.90 \pm 3.85$ \\
\hline $1-S N$ & $98.76 \pm 4.28$ & $99.33 \pm 4.30$ & $88.63 \pm 2.90$ \\
\hline $1-M P$ & $89.66 \pm 3.03$ & $90.33 \pm 2.93$ & \\
\hline
\end{tabular}


TABLE (2) Means and standard deviations (SD) of post treatment Cephalometric measurements changes for the three studied groups and results of Tukey test.

\begin{tabular}{|c|c|c|c|}
\hline \multirow{2}{*}{ Measurements } & $\begin{array}{c}\text { Group 1 } \\
\text { (rigid chin cup) }\end{array}$ & $\begin{array}{c}\text { Group 2 } \\
\text { (elastic chin cup) }\end{array}$ & $\begin{array}{c}\text { Group 3 } \\
\text { (control) }\end{array}$ \\
\cline { 2 - 4 } & Means \pm SD & Means \pm SD & Means \pm SD \\
\hline SNA & $0.19 \pm .40^{\mathrm{A}}$ & $0.23 \pm 0.43^{\mathrm{A}}$ & $0.18 \pm 0.40^{\mathrm{A}}$ \\
\hline SNB & $-.1 .57 \pm .50^{\mathrm{A}}$ & $-1.52 \pm 0.60^{\mathrm{A}}$ & $0.36 \pm 0.50$ \\
\hline ANB & $.1 .76 \pm .70^{\mathrm{A}}$ & $1.76 \pm 0.83^{\mathrm{A}}$ & $-0.18 \pm 0.75$ \\
\hline Wits & $3.47 \pm .67^{\mathrm{A}}$ & $3.71 \pm 1.05^{\mathrm{A}}$ & $1.45 \pm 0.40$ \\
\hline Ar-Me & $1.19 \pm .51^{\mathrm{A}}$ & $0.95 \pm 0.66^{\mathrm{A}}$ & $0.81 \pm 0.40$ \\
\hline Ar-Go & $-0.47 \pm 0.51^{\mathrm{A}}$ & $-0.61 \pm 0.66^{\mathrm{A}}$ & $0.81 \pm 0.40^{\mathrm{A}}$ \\
\hline Go-Me & $0.80 \pm 0.40^{\mathrm{A}}$ & $0.90 \pm .43^{\mathrm{A}}$ & $0.90 \pm 0.30^{\mathrm{A}}$ \\
\hline Ar-Go-Me & $0.71 \pm 0.46^{\mathrm{A}}$ & $0.95 \pm .49^{\mathrm{A}}$ & $1.45 \pm 0.68$ \\
\hline N-Me & $3.28 \pm 1.23^{\mathrm{A}}$ & $4.04 \pm 1.43^{\mathrm{A}}$ & $0.63 \pm 0.50$ \\
\hline SN-MP & $1.38 \pm 0.49^{\mathrm{A}}$ & $1.47 \pm 1.07^{\mathrm{A}}$ & $0.81 \pm 0.40^{\mathrm{A}}$ \\
\hline $1-\mathrm{SN}$ & $0.85 \pm 0.47^{\mathrm{A}}$ & $0.90 \pm 0.53^{\mathrm{A}}$ & $0.18 \pm 0.75$ \\
\hline $1-M P$ & $-2.28 \pm 0.90$ & $-3.61 \pm 1.07$ & -18 \\
\hline
\end{tabular}

Means with the same superscripted letters in each row are not significantly different at $P \leq 0.05$ according to the Tukey test.

TABLE (3) Skin of the chin evaluation index and the results of Chi-square test.

\begin{tabular}{|c|c|c|c|c|c|c|}
\hline \multirow[t]{2}{*}{ Index } & \multicolumn{2}{|c|}{$\begin{array}{l}\text { Soft chin cup } \\
\qquad \mathrm{N}=21\end{array}$} & \multicolumn{2}{|c|}{$\begin{array}{l}\text { Hard chin cup } \\
\qquad \mathrm{N}=21\end{array}$} & \multicolumn{2}{|c|}{$\begin{array}{c}\text { Control } \\
\mathrm{N}=11\end{array}$} \\
\hline & $\mathrm{N}$ & $(\%)$ & $\mathrm{N}$ & $(\%)$ & $\mathrm{N}$ & $(\%)$ \\
\hline 0 & 5 & (23) & 4 & (19) & 11 & 100 \\
\hline 1 & 13 & (61.9) & 10 & (47.7) & 0 & 0 \\
\hline 2 & 3 & (14.3) & 7 & (33.3) & 0 & 0 \\
\hline $\begin{array}{l}\text { Chi-square within each } \\
\text { group }\end{array}$ & \multicolumn{2}{|c|}{$\begin{array}{c}\mathrm{X}^{2}=8 \\
\mathrm{P}=0.018\end{array}$} & \multicolumn{2}{|c|}{$\begin{array}{c}\mathrm{X}^{2}=2.59 \\
\mathrm{P}=0.28\end{array}$} & & \\
\hline $\begin{array}{l}\text { Overall chi-square } \\
\text { Soft versus Hard }\end{array}$ & \multicolumn{2}{|c|}{$\mathrm{X}^{2}=28.8$} & & & \multicolumn{2}{|c|}{$\begin{array}{l}\mathrm{P}<0.001 \\
\mathrm{P}=0.34\end{array}$} \\
\hline
\end{tabular}

$0=$ no effects, 1 = redness, $3=$ laceration. 


\section{DISCUSSION}

In this study, equal amount of force was applied (300 g) through soft and hard chin cup in order to evaluate the effect of chin cup type/material on delivering the force to the chin. The he same force magnitude (300 g) In the treatment groups, there was a significant decrease in SNB angle compared to the untreated control group indicating a restraining mandibular growth action or/and a clockwise rotation of the mandible. The mandibular growth restriction after extraoral force application may result from the combination of morphologic changes in TMJ as well as remodeling of the mandible through apposition/resorption changes in the condyle. ${ }^{15}$ Meanwhile, the clockwise rotation of the mandible resulted in a significant increase in both anterior facial height (N-Me) and mandibular plane angle (SN-MP) in the treated groups. These findings are in line with earlier investigations. , $14,18,20,26^{2}$

A significant decrease in the mandibular ramus height (Ar-Go) was noticed in patients wearing chin cup despite its type compared to the control group. This reduction might be explained by the effect of posterior superior orthopedic force provided by occipital -pull head cap that induces pressure at the condylar superior border and alters the mandibular vertical growth. ${ }^{23,26}$ Similar findings were reported in previous studies. ${ }^{9,27}$ These findings were in contrast to those of other authors, who found an increase in ramus height and attributed it to the forward bending of the condylar head.$^{15,26,28}$ The mandibular length (Ar-Me) and gonial angle (Ar-Go$\mathrm{Me}$ ) exhibited no marked alterations between the treatment and the control group. These findings are in agreement with previous studies..$^{9,20,26,29}$ On the other hand, it was in contrast to that Altug et al. and others..$^{30,15,17}$ Such controversy could be attributed to the difference of chin cup treatment protocols or sample size and gender.

In this study, there was no significant changes in the mandibular corpus (Go-Me) between the three studied groups. This could be attributed to utilization of heavy forces for longer periods might be needed. In addition, it is impossible to load the entire condylar surfaces and the line of force is usually below the ideal one which may result in a more mandibular rotation than restricting mandibular growth. ${ }^{23}$ Ücüncü et al. reported similar results although Mitani and Fukazawo found significant retardation in the mandibular body length after using chin cup. ${ }^{26,28}$

Regarding the anteroposterior maxillary movement, SNA angle showed no difference between the treatment groups and the control one. A matched results were reported in former investigations. , $^{13,20,26,28}$ In contrast, Gokalp and Kurt verified that the utilization of chin cup led to a significant increase in the SNA. ${ }^{15}$ They referred the increase in the anteroposterior movement of the maxilla to the anterior cross bite correction. However, chin cup therapy extended for 19 months in their study compared to 12 months in the current study.

Patients treated with chin cup in this study expressed a significant improvement of class III relationship of the maxilla to the mandible proved by an increase in both ANB angle and Wits appraisal. The mandibular clock wise rotation and anterior facial height increase are together advantageous in class III treatment. Similar findings were reported in other researches. ${ }^{9,13,20,26,28}$ However, the greater increase reported with Altug et al. was reflecting the effect of personalised treatment philosophy developed in this study. ${ }^{30}$

The results also revealed a significant reduction in the inclination of the lower incisors in the two treatment groups in comparison to the control one. In addition, utilization of soft chin cup led to significantly more lingual inclination than the hard chin cup. This finding was the only difference between the two types of chin cup. Although chin cup applied the force to the chin rather than the teeth it migrates upward toward the lower lip pressing on 
the lower incisors leading to a lingual inclination of the lower incisors. This upward migration could be more with the soft chin cup could resulting in more pressure on the lower incisors and subsequently more lingual tipping than with the hard one. ${ }^{23}$

In this study, the authors developed the Skin Irritation Index to evaluate the effect of chin cup on the skin underneath. Results of the current study revealed that chin cup application either hard or soft was associated with skin irritation manifested by erythema and/or laceration (Table 3). Erythema was the dominant effect for either soft or hard chin cup. However, laceration (Figure 3) was greater in hard chin cup group and no significant differences were found between soft and hard chin cup. Interestingly, development of skin laceration in some patient was associated with negative reaction on patient cooperation. Patients those suffered from erythema or laceration was referred to the specialist and had the proper medication and instructed to quit wearing the appliance until healing occurred. However, the period of appliance removal to ensure proper healing of the skin underneath the chin cup did not exceed 14 days in both treatment groups. Hard chin cup did not have the similar contour of the chin and hence the force could be concentrated in certain areas resulting in more aggressive reaction of the skin. On the other hand, the soft chin cup follows the chin contour resulting in an equal distribution of the force over the chin.

\section{CONCLUSIONS}

Hard as well as soft chin cup are effective methods in the treatment of class III malocclusions. Both types of the chin cup have almost the same dental and skeletal effects. The soft chin cup produces more lingual tipping of the lower incisors. Erythema of the skin underlying the chin cup was the dominant effect of both types and lacerations were greater with hard chin cup than the soft one.

\section{REFERENCES}

1. Mitani H, Sato K, Sugawara J. Growth of mandibular prognathism after pubertal growth peak. Am J Orthod Dentofacial Orthop. 1993 Oct; 104(3):330-36.

2. Lee J, KimW, Suhr H. Study of recognition of malocclusion and orthodontic treatment. Korean J Orthod.1994 Mar; 24(1):367-94.

3. Ko Y, Baek H, Mah, J Yang S. Determination of successful chincup therapy in skeletal class III malocclusion. Am J Orthod Dentofacial Orthop. 2004 Jul; 126(1):33-41.

4. Pangrazio-Kulbersh V, Burger J, Kersten G. Effects of protraction mechanics on the midface. Am J Orthod Dentofacial Orthop. 1998 Nov; 114(5):484-91.

5. Kilic N, Celikoglu M, Oktay H.Effects of functional regulator III on profile changes in subjects with maxillary deficiency. Eur J Orthod .2010b May; 32(6):729-43.

6. Tang E L. The prevalence of malocclusion amongst Hong Kong male dental students. Br J Orthod .1994 Feb; 21(1):57-63.

7. McNamara JA Jr. An orthopedic approach to treatment of class III malocclusion in young patients. J Clin Orthod .1987 Sep; 21(9):598-608.

8. Thailander B and Myrberg N. The prevalence of malocclusion in Swedish schoolchildren. Scand J Dent Res.1973 Feb; 81(1):12-21.

9. Graber LW. Chin cup therapy in mandibular prognathism. Am J Orthod.1977 Jul; 72(1):23-41.

10. Cozza P, Marino A, Mucedero M. An orthopedic approach to the treatment of Class III malocclusion in the early mixed dentition. Eur J Orthod 2004 Apr; 26(2):191-99.

11. Baccetti T, Franchi L, McNamara J. Treatment and posttreatment craniofacial changes after rapid maxillary expansion and facemask therapy. Am J Orthod Dentofacial Orthop.2000 Oct; 118(4):404-13.

12. Giancotti A, Maselli A, Mampieri G, Spano` E. PseudoClass III malocclusion treatment with Balters' Bionator. J Orthod. 2003 Sept; 30(3):203-15.

13. Tuncer C, Uner O. Effects of a magnetic appliance in functional Class III patients. Angle Orthod. $2005 \mathrm{Sept}$; 75: 768-77.

14. Deguchi T, Kuroda T, Minoshima Y, Graber TM. Craniofacial features of patients with Class III 
abnormalities: growth related changes and effects of short-term and long-term chincup therapy. Am J Orthod Dentofacial Orthop. 2002 Jan; 121(1):84-92.

15. Gökalp H, Kurt G. Magnetic resonance imaging of the condylar growth pattern and disk position after chin cup therapy: a preliminary study. Angle Orthod. $2005 \mathrm{Jul}$; 75(4): 568-75.

16. Allen RA, Connolly IH, Richardson A. Early treatment of Class III incisor relationship using the chincap appliance. Eur J Orthod.1993 Oct; 15(5):371-76.

17. Barrett AA, Baccetti T, McNamara JA Jr .Treatment effects of the light-force chincup. Am J Orthod Dentofacial Orthop. 2010 Oct; 138(4):468-76.

18. Sugawara J, Mitani H. Facial growth of skeletal Class III malocclusion and the effects, limitations, and long-term dentofacial adaptations to chincap therapy. Semin Orthod. 1997 Dec; 3(4):244-54.

19. Sugawara J. Clinical practice guidelines for developing Class III malocclusion. In: Nanda R, editor. Biomechanics and Esthetic Strategies in Clinical Orthodontics. US: Saunders; 2005: p. 211-263.

20. Abdelnaby YL, Nassar EA. Chin cup effects using two different force magnitudes in the management of Class III malocclusions. Angle Orthod. 2010 Sept; 80(5):957-62.

21. Deguchi T, McNamara JA. Craniofacial adaptations induced by chincup therapy in Class III patients. Am J Orthod Dentofacial Orthop .1999 Feb; 115(2):175-82.

22. Tuncer BB, Kaygisiz E, Tuncer C, Yüksel S. Pharyngeal airway dimensions after chin cup treatment in Class
III malocclusion subjects. J Oral Rehabil .2009 Feb; 36(2):110-7.

23. Iida Y,Deguchi T, Kageyama T. Chin cup treatment outcomes in skeletal Class III dolicho-versus nondolichofacial patients. Angle Orthod .2005 Jul; 75(4):576-83.

24. Katashiba S, Deguchi Sr T, Kageyama T, Minoshima Y, Kuroda T, Roberts WE. The aggressive chin cup protocol (14 h/day for 2 years with excellent compliance) depends on commitment to overcorrection of the skeletal Class III malocclusion. Orthod Waves.2006 Jun; 65(2):57-63.

25. Bolognia J, Jorizzo J, Schaffer J. Dermatology. $3^{\text {rd }}$ ed. Elsevier, Saunders; 2012.

26. Proffit WR, Fields HW Jr, Sarver DM. Contemporary Orthodontics. 4th ed. St Louis, MO: Mosby; 2007.

27. Ritucci R, Nanda R. The effect of chincup therapy on the growth and development of the cranial base and midface. Am J Orthod Dentofacial Orthop .1986 Dec; 90(6):475-83.

28. Mitani H, Fukazawo H. Effects of chincap force on the timing and amount of mandibular growth associated with anterior reversed occlusion (Class III malocclusion) during puberty. Am J Orthod Dentofacial Orthop. 1986 Dec; 90(6):454-63.

29. Sakamoto T, Iwase I, Uka A, Nakamura S. A roentgenocephalometric study of skeletal changes during and after chin cap treatment. Am J Orthod .1984 Apr; 85(4):341-50.

30. Altuğ Z, Erdem D, Rübendüz M. Investigation of the functional treatment effects of the skeletal and dental Class III anomalies on the skeletal region. Ankara Üniversitesi Diş Hekimliği Fakültesi dergisi . 1989 Sept; 16(3):447-52. 